\title{
Pediatric COVID-19 in Sub-Saharan Africa: A Series of Cases Report in Cameroon
}

\author{
Mekone Nkwele Isabelle1,2*, Chelo David1, Eloundou Odi Eugene', Sap Ngo Um Suzanne1, \\ Meli Josephine ${ }^{2}$, Hadja Hamsatou' ${ }^{2}$ Balla Nkonda Alain ${ }^{3}$, Minka Basilice ${ }^{1}$, \\ Djientcheu Vincent de Paul1,2
}

\author{
${ }^{1}$ Faculty of Medicine and Biomedical Sciences, Department of Pediatrics, University of Yaoundé I, Yaoundé, Cameroon \\ ${ }^{2}$ Yaoundé General Hospital, Yaoundé, Cameroon \\ ${ }^{3}$ Jordan Medical Service, Yaoundé, Cameroon \\ Email: `isamekone@yahoo.fr, chelodad6@yahoo.fr, dil1trou@yahoo.fr, suzysap@gmail.com,jmeli_cm@yahoo.fr, \\ basiobama@gmail.com, Hhamsou@yahoo.fr, ballankondaalain@gmail.com, vincent_djientcheu@yahoo.com
}

How to cite this paper: Isabelle, M.N., David, C., Eugene, E.O., Suzanne, S.N.U., Josephine, M., Hamsatou, H., Alain, B.N., Basilice, M. and de Paul, D.V. (2020) Pediatric COVID-19 in Sub-Saharan Africa: A Series of Cases Report in Cameroon. Open Journal of Pediatrics, 10, 363-368. https://doi.org/10.4236/ojped.2020.102037

Received: May 18, 2020

Accepted: June 26, 2020

Published: June 29, 2020

Copyright $\odot 2020$ by author(s) and Scientific Research Publishing Inc. This work is licensed under the Creative Commons Attribution International License (CC BY 4.0).

http://creativecommons.org/licenses/by/4.0/ (c) (i) Open Access

\begin{abstract}
Background: Infection with the new Coronavirus 2019 SARS-CoV-2, COVID-19, is a global pandemic that does not spare children. Pediatric forms of COVID-19 are increasingly described in the literature with less severe clinical forms and lower mortality as compared to adults. We do not have data on the epidemiologic characteristics of SARS-CoV-2 infection in children in sub-Saharan Africa where the pandemic is less severe. Aim: We present four cases of pediatric COVID-19 in Cameroun. Case Presentation: These cases of COVID-19 concern children aged 12 weeks to 13 years. We discuss the diagnostic difficulties and the issues of effective management in the context of a resource-constrained country. Conclusion: COVID-19 affects children in sub-Saharan Africa. There are different clinical presentations with favorable outcomes, but there is a need for greater vigilance in the pediatric population.
\end{abstract}

\section{Keywords}

COVID-19, Children, Cameroon, Sub-Saharan Africa

\section{Introduction}

The coronavirus disease 2019 (COVID-19), caused by Severe Acute Respiratory Syndrome Coronavirus 2 (SARS-CoV-2), spread around the world and currently reaches all continents with report of the first cases in China since December 2019. This condition, which manifests itself, in its symptomatic forms, by a set of respiratory and extra-respiratory clinical signs, affects all age groups [1]. As of 
April 26, 2020, the World Health Organization declared 2,804,796 confirmed cases and 122,218 deaths due to COVID-19 [2]. Current available data indicate a lower outbreak rate in children as compared to adults. On average, $1 \%$ to $2 \%$ of children and adolescents under the age of 19 are affected, less in-patient and intensive care of children who are positive for COVID-19 has been recorded [3] [4]. The symptoms of most reported pediatric cases of COVID-19 are those of a relatively benign viral infection [5]. In sub-Saharan Africa, where the pandemic is less severe, there is lack of data on the epidemiology of SARS-CoV-2 infection in children. This is a report of four clinical cases of children infected with SARS-CoV-2 having clinical manifestations of COVID-19 in Cameroon, a country with limited resources.

\section{Cases Presentation}

\section{Clinical Case 1}

This is that of a male infant, aged 12 weeks, with a non-peculiar perinatal history. He had arrived in Cameroon from a European country in an outbreak of COVID-19 with an asymptomatic mother tested positive. Brought to the pediatric emergency room for fever and cold, the clinical examination revealed a non-ill-looking general condition associated with a fever-free, rhino-bronchial catarrh and a positive nasal swab to COVID-19. He was put on observation in a hospital setting with his mother, nasal unclogging was done every 4 hours using physiological serum and he was breastfed more frequently. His test on the 14th day was negative and, given that the clinical examination was normal, the discharge was allowed.

\section{Clinical Case 2}

Female infant aged 22 months; without a contributing history, consulted for dyspnea and feeding difficulties evolving for 24 hours in a context of fever and cough that started 3 days before the onset of symptoms. The child consulted on the eve, did a chest $\mathrm{x}$-ray that was normal (Figure 1) and paracetamol was prescribed to her. Her father, returning to his wife and children, had flown back from a European country in an outbreak of COVID-19 the previous two weeks. He consulted 24 hours earlier with the same symptoms; his daughter had associated with generalized myalgia and was placed in hospital isolation where a nasal swab was carried out for SARS-CoV-2 by rtPCR. Upon arrival of the infant in the hospital they were all kept in a common isolation room for 4 days before the father's positive results were rendered as well as a chest scan revealing serious infection abnormalities to COVID-19. The mother was asymptomatic. The initial physical examination of the infant revealed: a non-ill-looking general state, fever, bilateral bronchial rales, and coarse crackles at the right basal lung field with no signs of acute respiratory distress. The chest scan found right middle lobar pneumonia (Figure 2). The full blood count results were as follows: Hb: 11.5 g/dl; MCHC: 31.2 g/dl; MCV: 76 FL leukocytes: 9.1 G/L; lymphocytes: 7.10 G/L; neutrophils $1.27 \mathrm{G} / \mathrm{L}$. 


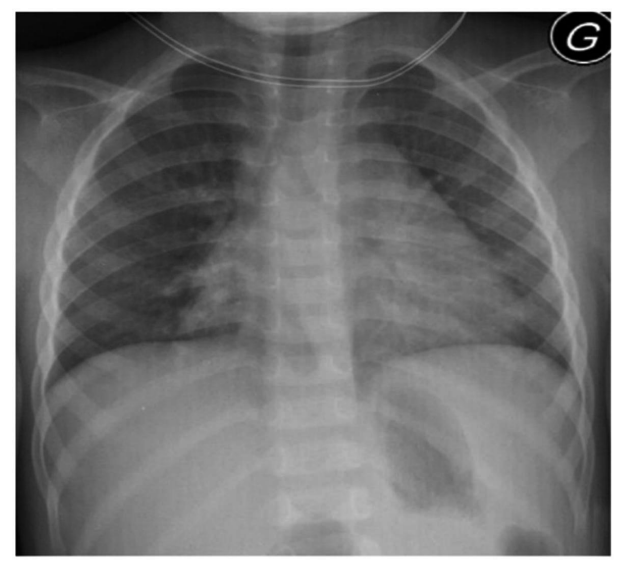

Figure 1. Antero posterior supine normal chest x-ray.

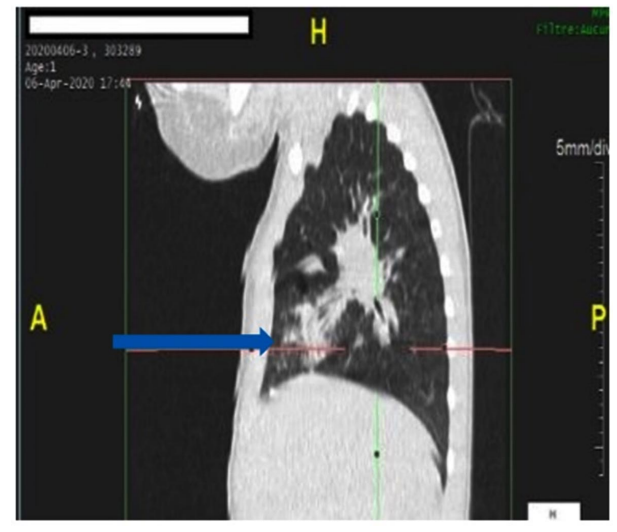

Figure 2. Right pulmonary Sagittal section showing a confluent opacity with air broncho gram (arrow).

The rest of the laboratory tests found: CRP $<6 \mathrm{mg} / \mathrm{l}$; urea: $0.2 \mathrm{~g} / \mathrm{l}$; creatinemia: $9.71 \mathrm{mg} / \mathrm{l}$; SGOT: $48.23 \mathrm{UI} / \mathrm{l}$; SGPT: $14.58 \mathrm{UI} / \mathrm{l}$; Blood group: O positive; HIV: negative; $\mathrm{Hb}$ electrophoresis: AA. She was hydrated and given an antibiotherapy (ceftriaxone: $50 \mathrm{mg} / \mathrm{kg} / \mathrm{d} \mathrm{IV}$; gentamycin: $5 \mathrm{mg} / \mathrm{kg} / \mathrm{d} \mathrm{IV}$; azithromycin 20 $\mathrm{mg} / \mathrm{kg} / \mathrm{d} \mathrm{PO}$ ), an antimalarial treatment (Association of artesunate $100 \mathrm{mg}+$ mefloquine $95 \mathrm{mg}$ single daily dose for 3 days), Zinc $20 \mathrm{mg} / \mathrm{d}$ (PO). The nasal swab for COVID-19 screening by rtPCR was done on the 4th day of the in-patient care. On the 5th day there was no fever, a regression of cough and better nutrition. The result of the rtPCR was negative whereas that of the mother was positive without her having any symptoms of coronavirus disease. The child was discharged on the 10th day of hospitalization with a very favorable clinical evolution.

\section{Clinical Cases 3 and 4}

This is the case of two sisters aged 3 and 13 years old. They were tested positive to COVID-19 as well as their parents as contact subjects of an adult returning from an epidemic European country, who had severe respiratory symptoms and was tested positive for COVID-19. Both girls remained asymptomatic during the 14 days of hospital isolation and received clinical surveillance. They 
could return to family after their control tests were negative on the 14th day.

A careful clinical follow up of these four patients was initiated to early identify complications.

\section{Discussion}

This is the report of four cases of COVID-19 in children aged 12 weeks to 13 years old. It is particularly important to study this disease in children because they are more likely to be asymptomatic carriers, but also because some of them can develop severe forms with high risk of death [5] [6]. The infection of children of varying ages confirms that COVID-19 affects all pediatric age groups. However, a review of the literature on the epidemiology of pediatric shows that forms prevalence, morbidity, and mortality are far below those of adults [6] [7]. The manual and airborne modes of transmission make the pathology difficult to avoid and cohabitation without barrier measures with an infected person makes it almost inevitable for contacts to be contaminated. This explains while three of the children in the series were infected by their entourage.

The nasal swab rtPCR is the biological test used in Cameroon to diagnose COVID-19. This is a test with excellent specificity when the sampling is correctly carried out though it has a reduced sensitivity [8]. As a result, it is of interest to sometimes repeat it or associate it with a serological test. This was not done to the 22-month-old infant who was taken care of, as the tests were unavailable. Thus, the test result was negative for an infant subject to a risk of contamination as both parents were infected with SARS-CoV-2, having obvious respiratory symptoms, radiological and biological abnormalities, as described in other series of cases. Repeating this rtPCR test on other nasopharyngeal samples or stools or its association with a serological test would likely have increased the chances of diagnosing COVID-19 [8].

It should be noted that the infant who had a highly symptomatic clinical form, and whose evolution was good, was managed, by combining azithromycin, zinc and an antimalarial drug containing mefloquine. The other children did not receive specific treatments and their clinical outcome was favorable. It is important to establish in children the indications of treatment by considering the availability of pediatric forms and their physiological specificities [9]. Hydroxy chloroquine is used in adult therapeutic protocols in Cameroon, but its administration in children raises the problem of tolerance and resistance development associated with the use of antimalarials in sub-Saharan Africa in age groups where malaria prevalence is very high [10].

No deaths were reported in this series of cases, but child deaths due to COVID-19 have been recorded worldwide since the beginning of the pandemic and these are more frequent in countries with high prevalence [4]. More analyses about the current cases were needed, and due to lack of resources, they could not be done. A greater sample of similar cases can be followed up and reviewed.

It is important to have broader and more accurate epidemiological data on 
COVID-19 in children in sub-Saharan Africa where access to care services is sometimes limited and misdiagnosis more frequent. This will provide real mapping of the clinical forms of COVID-19 in the pediatric population, refine diagnostic methods, and establish effective therapeutic protocols. Better yet, the majority of these children living in poverty and in an environment with potentially hazardous sanitary conditions could be the key to understanding the effects of the disease by looking into the composition of their intestinal microbiota [11] [12].

\section{Conclusion}

With this description of 4 cases, we want to draw attention to COVID-19 infection in our pediatric environment. Our experience shows that all ages are concerned and that the clinical pictures are polymorphic, ranging from totally asymptomatic cases to pneumonia which can be severe. The evolution of the cases has been good. There is a necessity for greater vigilance in the pediatric population, where anamnesis in search of contributive history must be a key element in the search for a diagnosis. In addition, health care personnel in charge of children in this epidemic period should protect themselves against possible contamination from patients, regardless of the symptoms.

\section{Acknowledgements}

We thank the parents of the different children for allowing us to publish their cases.

\section{Conflicts of Interest}

The authors declare no conflicts of interest regarding the publication of this paper.

\section{References}

[1] Tu, Y.F., Chien, C.S., Yarmishyn, A.A., et al. (2020) A Review of SARS-CoV-2 and the Ongoing Clinical Trials. International Journal of Molecular Sciences, 21, 2657. https://doi.org/10.3390/ijms21072657

[2] World Health Organization. Coronavirus disease 2019 (COVID-19): Situation Report-97.

[3] Wu, Z. and McGoogan, J.M. (2020) Characteristics of and Important Lessons from the Coronavirus Disease 2019 (COVID-19) Outbreak in China: Summary of a Report of 72314 Cases from the Chinese Center for Disease Control and Prevention. JAMA, 323, 1239-1242. https://doi.org/10.1001/jama.2020.2648

[4] Lugvigsson, J.F. (2020) Systematic Review of COVID-19 in Children Shows Milder Cases and a Better Pronosis than Adults. Acta Paediatrica, 109, No. 6. https://doi.org/10.1111/apa.15270

[5] She, J., Liu, L. and Liu, W. (2020) COVID-19 Epidemic: Disease Characteristics in Children. Journal of Medical Virology, 92, No. 7. https://doi.org/10.1002/jmv.25807

[6] Lu, X., Zhang, L., Du, H., et al. (2020) SARS-CoV-2 Infections in Children. The New England Journal of Medicine, 382, 1663-1665. 
https://doi.org/10.1056/NEJMc2005073

[7] Lee, P.I., Hu, Y.L., Chen, P.Y., et al. (2020) Are Children Less Susceptible to COVID-19? Journal of Microbiology, Immunology and Infection, 53, 371-372. https://doi.org/10.1016/j.jmii.2020.02.011

[8] Loeffelholz, M.J. and Tang, Y.W. (2020) Laboratory Diagnosis of Emerging Human Coronavirus Infections-The State of the Art. Emerging Microbes \& Infections, 9, 747-756. https://doi.org/10.1080/22221751.2020.1745095

[9] Zhai, P., Ding, Y., Wu, X., et al. (2020) The Epidemiology, Diagnosis and Treatment of COVID-19. International Journal of Antimicrobial Agents, 55, 105955. https://doi.org/10.1016/j.ijantimicag.2020.105955

[10] World Health Organization (2019) World Malaria Report.

[11] Kortekangas, E., Young, R., Cheung, Y.B., Fan, Y.M., Jorgensen, J.M., Kamng'ona, A.W., et al. (2019) A Prospective Study on Child Morbidity and Gut Microbiota in Rural Malawi. Journal of Pediatric Gastroenterology and Nutrition, 69, 431-437. https://doi.org/10.1097/MPG.0000000000002435

[12] Mello, C.S., Carmo-Rodrigues, M.S., Filho, H.B., et al. (2016) Gut Microbiota Differences in Children from Distinct Socioeconomic Levels Living in the Same Urban Area in Brazil. Journal of Pediatric Gastroenterology and Nutrition, 63, 460-465. https://doi.org/10.1097/MPG.0000000000001186

\section{Abbreviations}

SARS: Severe Acute Respiratory Syndrome

COV: Coronavirus

COVID-19: Coronavirus Disease 2019 